\title{
Evaluation of tomographic brain reconstruction techniques: case of the shepp-logan phantom
}

\begin{abstract}
Carcinogenic tumors are the leading cause of death in the world. When clinicians have excellent shot, they are able to detect a tumor early and eradicate it easily by chemotherapy or by simple surgery. We propose in this paper to compare different methods of tomographic reconstruction of the brain from the point of view of the quality of the image provided, in order to propose to the physicians the algorithms which guarantee them the best image results. We use two comparison metrics based on the PSNR (Peak Signal to Noise Ratio) and the HVS (Human Visual System). The association of these two objective and subjective criteria show that the methods which exploit the reconstruction of the samples by subset provide images of better quality.
\end{abstract}

Volume 2 Issue 6 - 2017

\author{
Emmanuel Tonye, Ivan Kabiena
}

University of Yaounde I, Cameroon

Correspondence: Emmanuel Tonye, National Advanced School of Engineering, Yaounde, Cameroon; Email tonyee@hotmail.com

Received: January 09, 2017| Published: June 19, 2017

Keywords: tumors, cancer, brain, image, quality, tomography, SVH, PSNR

Abbreviations: PSNR, peak signal to noise ratio; HVS, human visual system; Fig, figure; SIRT, simultaneous iterative reconstruction technique; ART, algebraic reconstruction technique; ART-DS, algebraic tomographic reconstruction by selective discrimination; SIRT-DS, simultaneous iterative reconstruction technique by selective discrimination; ITU, international telecommunication union; SSCQS, single stimulus continuous quality scale; IC, confidence interval; MOS, mean opinion score

\section{Introduction}

Positron emission tomography is based on the reconstruction of sections of the interior of the human body after injection or ingestion of an active radio isotope. This radioactive isotope, generally the ${ }^{18} \mathrm{~F}$ will interact with the human cells specific to the organ we wish to observe, and emit two diametrically opposed gamma $(\gamma)$ photons. ${ }^{1}$ A detection ring is then used to detect these photons (Figure 1). ${ }^{2}$ By measuring a certain number of photon pairs, it will be possible, via mathematical techniques, to obtain an image estimating the overall distribution of the emission sites of these photons. Several reconstruction techniques exist in the literature: analytical methods and iterative methods. ${ }^{3-5}$ The deterministic iterative methods which allow a better quantification of the noise have been implemented in the framework of this work, the most successful methods are the ART method ${ }^{6-7}$ in its variants ARTDS, ${ }^{7}$ kaczmarz, ${ }^{8}$ Sym-Kaczmarz, ${ }^{9}$ and the SIRT method $^{10}$ in its SIRT$\mathrm{DS}^{11}$ and Cimmino ${ }^{10}$ variants. These iterative methods consist in the correction of the reconstructed image $f$ by successive iterations. At each iteration $k$, the error between $f^{(k)}$ and $f^{(k-1)}$ must be as small as possible in order to allow the convergence of the algorithm.

\section{General principle of ART methods}

The mathematical expression of the correction according to the ART (Algebraic Reconstruction Technique) method is easily obtained from the equation of the projection of a point on a hyperplane (Figure 2):

$$
f_{i}^{(k)}=f_{i}^{(k-1)}+R_{j i} \frac{p_{j}-R_{j} f^{(k-1)}}{R_{j}^{2}}
$$

This equation is interpreted as follows: each component $i$ of the vector $f^{(k)}$ at the iteration $k$ is corrected by adding to the value $f_{i}{ }^{(k-}$ 1) obtained at the preceding iteration a coefficient which is 0 if the Radius $j$ used for correction does not cross point $x_{\mathrm{i}}$ (in this case $R_{j i}$ is 0 ) and is otherwise proportional to the difference between the data $p_{j}$ (the true projection) and the projection recalculated from $f^{(k-l)}$ equal to $R f$ ${ }^{(k-1)}$. The normalization coefficient $\left\|\mathrm{R}_{\mathrm{j}}\right\|^{2}$ is the norm of the row of the matrix R corresponding to the data $j$, that is to say in the simple case considered here it is equal to the number of pixels traversed by the ray $j$. In short, the radius $j$ makes it possible to correct all the pixels that it traverses. At each iteration, a different ray is chosen, for example according to the following rule:

$$
j=k[m] . m \in I N(2)
$$

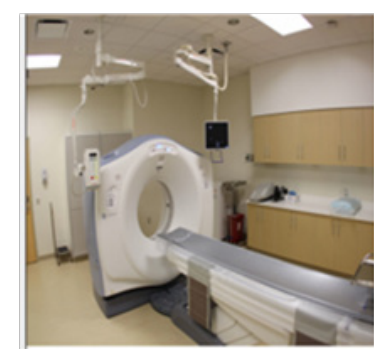

(a)

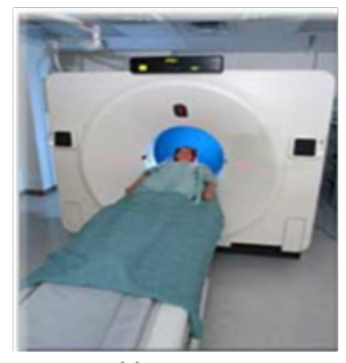

(b)
Figure I

(IA): Real tomograph, clinical practice of tomographic examination.

(IB): Source: Military Hospital of Val de Grace.

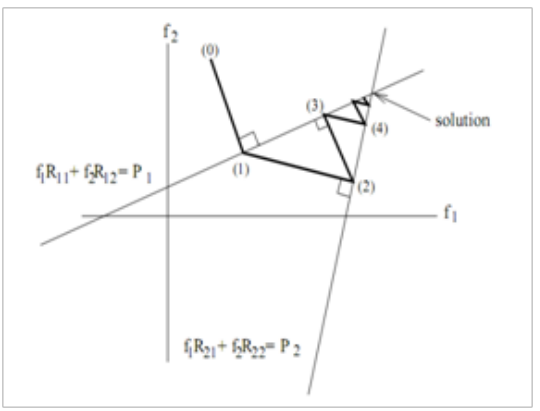

Figure 2 Principle of ART Methods. 
When the system $p=R f$ admits at least one solution, the algorithm converges to the minimum variance solution when it is initialized to 0 . On the other hand, when the data is noisy, the system may have no solution and a phenomenon of oscillations occurs.?

\section{General principle of SIRT methods}

The SIRT (Simultaneous Iterative Reconstruction Technique) method consists in performing the correction of each $f_{i}$ by using all the ray passing through $x_{i}$ (Figure 3 ). The equation for evaluating $f^{(k)}$ by correction of $f^{(k-1)}$ is as follows:

$$
f_{i}^{(k)}=f_{i}^{(k-1)}+\frac{\sum_{j} p_{j}}{\sum_{j} \sum_{i} R_{j i}}-\frac{\sum_{j} R_{j} f^{(k-1)}}{\sum_{j} R_{j}^{2}}
$$

The summation covering all the indices $j$ such that the radius $j$ crosses the pixel $x_{i}$. This method is slower than ART and requires more memory. ${ }^{10}$ The variants of these two methods involve for some a relaxation parameter (sym-kaczmarz, cimmino), and for the others a reconstruction of the $f_{i}$ in subsets (ART-DS, SIRT-DS).

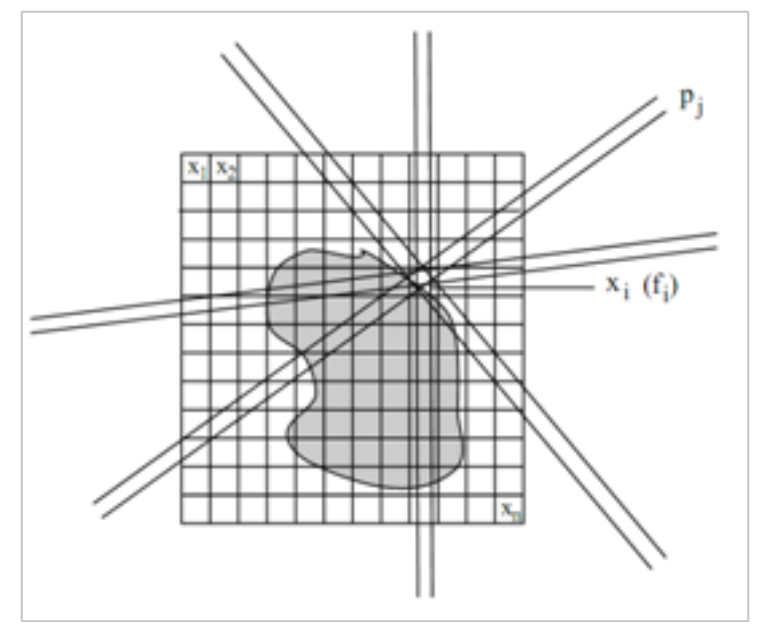

Figure 3 Principle of the SIRT method.

\section{Methods of comparison}

To evaluate qualitatively the capacity of each of these different variants to faithfully reconstruct a cut, let's reconstruct a section of the human brain. To do this, we will use the Shepp-Logan phantom (Figure 4), a digital image of synthesis used in tomographic research to model the brain. ${ }^{12}$ We will then use an objective criterion (PSNR) and a subjective criterion based on the human visual system (SVH), to compare the performances of the different methods.

Objective criterion: The PSNR: This type of metric is very widely used to numerically quantify the quality of reconstruction of an image. The signal-to-noise peak evaluates the distortion between the desired image and that reconstructed by the formula:

$$
P S N R=10 \log _{10} \sum_{(x, y)}\left(\frac{(g(x, y)-f(x, y))^{2}}{D}\right)
$$

Where $D$ is the image definition domain, $f(x, y)$ the pixel of coordinates $(x, y)$ of the reference image, and $G(x, y)$ the pixel of coordinates $(x, y)$ of the processed image. The effectiveness of this metric is only assured when it is associated with a subjective criterion based on validation by the human eye. ${ }^{13}$ In our results, we will use Student's statistical test ${ }^{14}$ for matched samples to show that there is a significant difference between the methods presented. The Student test for matched observations is used to compare the mean of two populations, each element of one of the populations being related to one element of the other. For example, it is possible to compare two treatments, the data being considered as pairs of observations (first observation of the pair receiving treatment 1 and second observation receiving treatment 2$)$. Let $\mathrm{x}_{\mathrm{ij}}$ be the observation $\mathrm{j}$ for the pair $\mathrm{i}(\mathrm{j}=1,2$ and $i=1,2, \ldots, n)$. For each pair of observations the difference $d_{i}=x_{i 2}$ $\mathrm{x}_{\mathrm{i} 1}$ is calculated. The statistical test is defined by:

$$
t_{o b s}=\frac{\bar{d}}{\sqrt{\frac{S_{d}^{2}}{n}}}
$$

Where $\mathrm{n}$ is the number of pairs of observations, $\mathrm{d}$ is the average of the differences between observations, and $\mathrm{S}_{\mathrm{d}}{ }^{2}$ the variance. More details on the use of this test are presented in. ${ }^{14}$

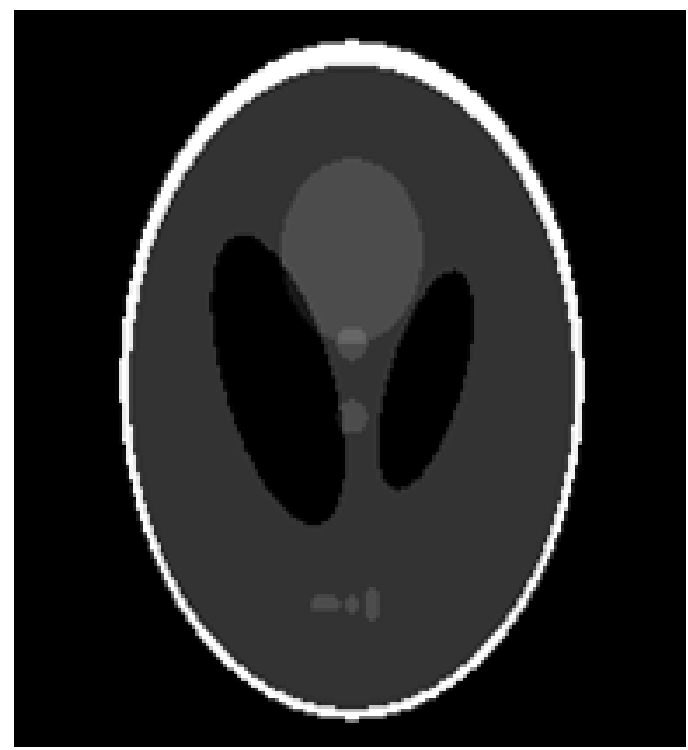

Figure 4 Cutting of the Shepp-Logan brain with the presence of a tumor.

Subjective criterion: simple stimulus protocol: Considered as the most reliable means of measuring the real quality, the subjective evaluation calls directly to the human observer. The human observer is required to judge the quality of the images presented to him according to a grid of evaluation with several levels of appreciation. At the end of these tests, a subjective score called MOS (Mean Opinion Score) is obtained. When carrying out these tests, it is important to pay particular attention to certain factors that may affect the participant's judgments, such as: observation distance, viewing conditions such as surrounding lighting, Screen calibration, image selection, psychological factors, and the nature of the observers. Different subjective assessment methods have been defined by the ITU (International Telecommunication Union). ${ }^{15}$ There are basically three main families: simple stimulus tests to judge image quality without any information about the original image. Dual stimulus tests where the observer is asked to judge the similarity between two images and the comparative tests whose main objective is to compare 
two or more stimuli. We present in the following the simple stimulus protocol that was chosen for this exercise, given its approximation with the clinical context of our experiment.

The Single Stimulus Continuous Quality Scale (SSCQS) method allows one to judge the quality of one stimulus at a time (without reference). It should be noted that the mode of presentation, illustrated in Figure 5, is thus limited. The images are presented one by one with a latency time between two presentations. This time allows the observer to assess the quality of the image. Scales with $5,6,7$ or 100 levels of appreciation can be used. Table 1 presents an example of a scale which is widely used to validate a quantitative or objective metric.

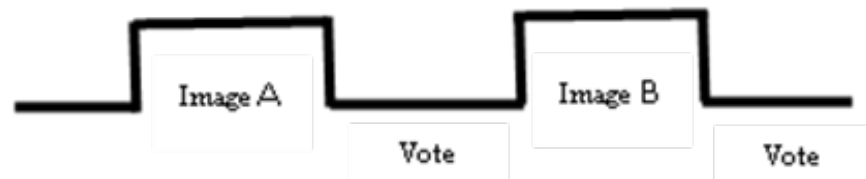

Figure 5 Single Stimulus Method.

Table I Example of rating scale

\begin{tabular}{lll}
\hline \multicolumn{2}{l}{ Five-point scale quality } \\
\hline Quality & \multicolumn{2}{l}{ Degradation } \\
\hline Excellent & 5 & Imperceptible \\
Good & 4 & Perceptible but not annoying \\
Fairly good & 3 & Slightly annoying \\
Poor & 2 & Embarrassing \\
Bad & I & Very annoying \\
\hline
\end{tabular}

\section{Discussion}

To evaluate our results, we will apply an objective measure based on the PSNR value obtained for each method and then the human eye will be used as a subjective measure in order to validate the objective measure. The experiments were carried out on a computer with two processors and the software MATLAB 7.5.0342 (R2007b) was used to program and compile our algorithms.

\section{Objective evaluation}

We have applied to the image of the human brain of Figure 2 the methods ART-DS, SIRT-DS, Kaczmarz, Sym-kaczmarz, Cimmino, over a noise range of 0 to $15 \%$ in $0.5 \%$ steps. The evolution of the PSNR as a function of the noise is given by Figure 6: As first observation, it emerges that the PSNR decreases with the increase of the noise. It is also noted that over all this noise range, the curves of the SIRT-DS and ART-DS methods are on top of the others, demonstrating that the quality of the reconstructed image is better with these two methods. This difference is significant from 0 to $2 \%$ and from 4 to $15 \%$ of noise. For a noise level of $5 \%$, Figure 7 shows the image of the corresponding brain reconstructed by the above methods:

Figure 7 (A) shows the cut of the desired brain, without noise, with the presence of a tumor in red. At a noise level $5 \%$, the tumor remains detectable (weakly) in the brain for the ART-DS (b) methods at 24.8 $\mathrm{dB}$ and SIRT-DS (c) at $21 \mathrm{~dB}$, but much less visible for the methods Kaczmarz (d) at $18.1 \mathrm{~dB}$ and sym-kaczmarz (e) at $15.5 \mathrm{~dB}$. Since the Cimmino method has the worst results of the objective criterion, it will not be used in the subsequent tests. The evolutionary curve of the
PSNR of the SIRT-DS method is below that of the ART-DS method, while for the other methods it is the kaczmark approach that is at the top. Also, by applying the student test between the SIRT-DS and kaczmarz methods, we can also conclude on the consistency of the difference between the ART-DS, SIRT-DS techniques and the other methods. Also, by varying the noise from 0 to $15 \%$ in steps of $0.5 \%$, the number of samples is greater than or equal to 30 . Table 2 shows the student test results between the SIRT-DS and kaczmarz methods.

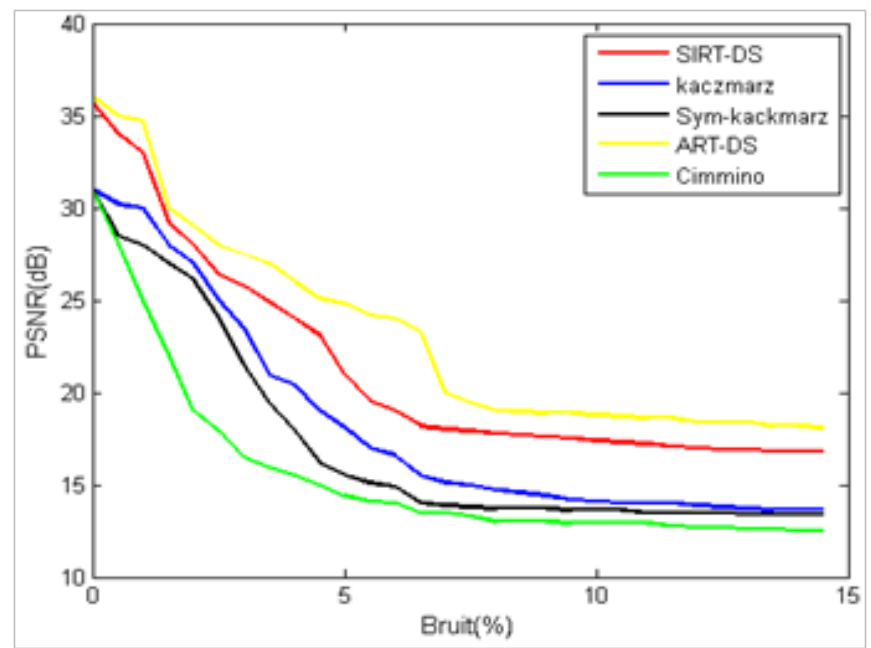

Figure 6 Evolution of the PSNR as a function of the noise for different approaches.

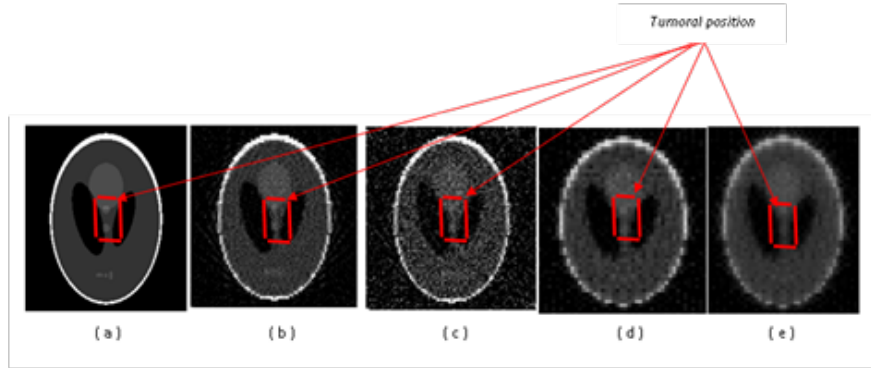

Figure 7 Cut of a non-noisy brain (A) and reconstructed at $5 \%$ level of noise by methods ART-DS (B), SIRT-DS (C), kaczmarz (D) and sym-kaczmarz (E), with presence of tumor.

It appears that the SIRT-DS method is better than the Kaczmarz method. Indeed, the mean number of PSNR for the SIRT-DS method $(21,29 \mathrm{~dB})$ is higher than that obtained for the Kaczmarz method $(18,29 \mathrm{~dB})$. The Student test for the comparison of two means from two matched samples shows that there is a significant difference between the mean numbers of PSNR for these two methods. It is therefore generally understood that the ART-DS and SIRT-DS techniques are better than the other methods in terms of the quality of the image solution provided.

\section{Subjective evaluation}

We have applied the simple stimulus protocol to a population of 50 individuals (doctors). Participants with pathologies of the eye were excluded. The images have been received on their mobile phones and they have been recommended to observe the following guidelines in order to obtain the most reliable evaluation possible:

i. Keep a minimum distance of $40 \mathrm{~cm}$ between the image and the eye; 
ii. Performing the test in a room with a single window to limit lighting;

iii. Apply initial configuration for telephone lighting;

iv. Space out the tests at least one hour;

v. Make the tests in the morning to avoid the stress of the work.

For reconstructed cuts of the brain, the observers had to attribute a note to the image between 1 and 5 according to the grid of appreciation described in Table 1, for different Algorithms (ART-
DS, SIRT-DS, Kaczmarz, Sym-Kaczmarz, the Cimmino method will not be used in this test given the results it provided in the objective assessment) at three distinct noise levels (without, $2 \%, 5 \%$ ); Table 3 presents the mean score (MOS) as well as the $95 \%$ confidence interval (IC) associated with this mean for each method and for a given noise level. Without additive noise, the mean scores of the ART-DS and SIRT-DS methods resulting from the subset treatments are higher than the mean scores of the Kaczmarz and Sym-kaczmarz methods which obtain similar scores and intervals of confidence almost identical to one another. At this level of noise, the vast majority of the participants manage to locate the tumor zone.

Table 2 Comparison of the PSNR of the SIRT-DS and Kaczmarz methods for the Logan phantom

\begin{tabular}{lllllll}
\hline \multicolumn{1}{l}{ Characteristics of variables } & & & & Student test for matched samples \\
\hline Variables & Minimum & Maximum & Average & Standard deviation & $|\mathrm{T}|$ & $q\left(1-\frac{0,05}{2}\right)(29)$ \\
\hline PSNR SIRT-DS (dB) & 16,80 & 35,70 & 21,29 & 5,75 & 21,21 & 2,045 \\
PSNR Kaczmarz (dB) & 13,60 & 31 & 18,29 & 5,83 & & \\
\hline
\end{tabular}

$|\mathrm{t}|$ :Absolute value of the Student test for matched samples; ddl: degree of freedom; $\left.q_{(m)}(n)\right)$ : Order quantile $m$ at $\mathrm{n}$ ddl.

Table 3 Mean score and associated IC for each method at different noise levels

\begin{tabular}{lllllll}
\hline \multirow{2}{*}{ Method } & \multicolumn{2}{l}{ Without additive noise } & \multicolumn{2}{l}{ With $\mathbf{2 \%}$ of additive noise } & \multicolumn{2}{l}{ With $\mathbf{5 \%}$ of additive noise } \\
\cline { 2 - 7 } & Mean score & IC à 95\% & Mean score & IC à 95\% & Mean score & IC à 95\% \\
\hline ART-DS & 4,24 & {$[4,04 ; 4,44]$} & 3,74 & {$[3,58 ; 3,90]$} & 2,92 & {$[2,72 ; 3,12]$} \\
SIRT-DS & 4,08 & {$[3,85 ; 4,30]$} & 3,5 & {$[3,34 ; 3,66]$} & 2,5 & {$[2,25 ; 2,75]$} \\
Kaczmarz & 3,96 & {$[3,75 ; 4,17]$} & 3 & {$[2,79 ; 3,20]$} & I,94 & {$[1,71 ; 2,17]$} \\
Sym-Kaczmarz & 3,98 & {$[3,75 ; 4,20]$} & 2,96 & {$[2,80 ; 3,12]$} & I,78 & {$[1,57 ; 1,98]$} \\
\hline
\end{tabular}

With additive noise of the order of $2 \%$, the difference between the ART-DS, SIRT-DS and Kaczmarz, Sym-kaczmarz methods becomes most important. The ART-DS and SIRT-DS approaches keep the best MOS, while the first difficulties of interpretations arise for the Sym-kaczmarz method which obtains a MOS of 2.96. At $5 \%$ of additive noise, which represents the maximum noise threshold for our experiment, the MOS show as a whole that for all approaches, the degradation is perceptible. However, the ART-DS and SIRT-DS methods give rise to a tolerance for high noise levels. It emerges that whatever the level of noise considered, the Shepp-Logan phantom representing a section of the brain with tumor, reconstructed by the Methods ART-DS and SIRT-DS receive on average better assessments.

\section{Conclusion}

We have demonstrated in this paper, starting from the exploitation of two objective and subjective criteria, that it is judicious for oncologists who give priority to good quality images, to opt for methods based on subset reconstruction like SIRT-DS and ART-DS, instead of other deterministic iterative methods, since they make it possible to detect start of tumors in the encephalon at high levels of noise. However, it is still necessary to validate these results in clinical practice on real subjects.

\section{Acknowledgements}

None.

\section{Conflict of interest}

The author declares no conflict of interest.

\section{References}

1. Pagani M, Stone Elander S, Larsson SA. Alternative positron emission tomography with non-conventional positron emitters: effects of their physical properties on image quality and potential clinical applications. Eur J Nucl Med. 1997;24(10):1301-1327.

2. Dreuille $\mathrm{O}$, Maszelin $\mathrm{P}$, Foehrenbach $\mathrm{H}$, et al. Principe et technique de la tomographie par émission de positons (TEP). 2002. $22 \mathrm{p}$.

3. Xu XL, Liow JS, Strother SC. Iterative algebraic reconstruction algorithms for emission computed tomography: A unified framework and its application to positron emission tomography. Med Phys. 1993;20(6):1675-1684.

4. Brooks RA, Di Chiro G. Principes of computer assisted tomography in radiographic and radio isotope imaging. Phys Med Biol. 1976;21(5):689732.

5. Dubois F. Reconstruction des images tomographiques par rétroprojection filtrée. Rev ACOMEN. 1998;4(2):92-99.

6. Zi-Jing Lin, Haijing Niu, Lin Li, et al. Volumetric diffuse optical tomography for small animals using A CCD-camera-based imaging system. International Journal of Optics. 2012. 10 p.

7. Kabiena IB, Tonye E, Ntsama PE. Algebraic tomographic reconstruction by selective discrimination ART-DS. American Journal of Signal Processing. 2013;3(4):106-112. 
8. Popa C. contrained kaczmarz extended algorithm for image reconstruction. Linear Algebra Appl. 2008;429(8-9):2247-2267.

9. Tommy E, Per Hansen C. Semi-convergence properties of kaczmarz's method. Inverse Problem. 2012;30(5).

10. Tommy E, Per Hansen C. Semi-convergence and relaxation parameters for projected SIRT. Journal of Scientific Computing. 2014;34(4):18.

11. Spitzbarth M, Drescher M. Simultaneous iterative reconstruction technique by selective discrimination SIRT-DS. International Journal of Innovative Research in Science Engineering and Technology. 2015;4(10):10367-10375
12. Shepp L, Logan BF. The Fourier reconstruction of a head section. IEEE Trans Nucl Sci. 1974;21(3):21-43.

13. Yusra AY Al Najjar, Der Chen Soong. Comparison of image quality assessment: PSNR, HVS, SSIM, UIQI. International Journal of Scientific \& Engineering Research. 2012;3(8):2229-5518.

14. Fourdrinier D. statistique inférentielle. Sciences sup. Mathématiques. 2002; $1: 358$.

15. Durant. Méthodes d'évaluation subjective de la qualité audiovisuelles pour applications multimédias, recommandation UIT-T. 1996. 911 p. 